\title{
Stoichiometry Issues in Pulsed Laser Deposition of Alloys Grown from Multicomponent Targets
}

\section{Citation}

Arnold, Craig B. and Michael J. Aziz. 1999. Stoichiometry issues in pulsed laser deposition of alloys grown from multicomponent targets. Applied Physics A 69(7). S23-S27.

\section{Published Version}

http://dx.doi.org/10.1007/s003390051349

\section{Permanent link}

http://nrs.harvard.edu/urn-3:HUL.InstRepos:2797292

\section{Terms of Use}

This article was downloaded from Harvard University's DASH repository, and is made available under the terms and conditions applicable to Other Posted Material, as set forth at http:// nrs.harvard.edu/urn-3:HUL.InstRepos:dash.current.terms-of-use\#LAA

\section{Share Your Story}

The Harvard community has made this article openly available.

Please share how this access benefits you. Submit a story.

Accessibility 


\title{
Stoichiometry Issues in Pulsed Laser Deposition of Alloys Grown from Multicomponent Targets
}

\author{
Craig B. Arnold" and Michael J. Aziz \\ Division of Engineering and Applied Sciences, \\ Harvard University, Cambridge, USA
}

\begin{abstract}
:
We are examining the degree of congruent transfer in Pulsed Laser Deposition (PLD) of alloy thin films in phases that are stable over a wide range of compositions. SiGe films were deposited by PLD on high purity glassy carbon substrates. We analyzed the average composition of these films using Rutherford backscattering spectrometry (RBS) and results show that the deposited films have a higher relative concentration of Ge than the initial targets. We infer that the non-congruent transfer is due to differential scattering in the plume itself. Additionally, the local composition of the particulates was measured using microprobe analysis and these observations are compared between a sintered target of $\mathrm{Si}$ and Ge powders and a solidified target of melted Si and Ge. The sintered target produces particulates with a wide range of compositions, whereas the solidified target produces five times fewer particulates with a tighter distribution of compositions. In contrast to the average composition of the films, the average composition of the particulates is the same as that of the targets. These results are discussed in terms of the microstructure of the targets and the melting process at the surface. The implications of these observations for composition determination by laser ablation are discussed.
\end{abstract}

One of the major advantages of Pulsed Laser Deposition (PLD) is the ability to transfer material stoichiometrically from a multicomponent ablation target to a growing film [1]. However, it is well known that such favorable results do not occur under all experimental conditions. Systems ranging from simple two-component metallic systems $[2,3]$ and semiconductor systems [4], to more complicated superconducting oxides [5] and ferroelectrics [6] have shown non-stoichiometric transfer of the target composition. The majority of the literature focuses on techniques to overcome this obstacle. For example, by controlling experimental parameters like the gas overpressure [7] or laser fluence [8,9], one can congruently transfer the composition of the target to the substrate.

An additional concern in PLD growth of materials is the presence of larger clusters or particulates in the growing films. Such particulates are normally deleterious to the resulting film properties, and most of the literature concentrates on methods to reduce the density of these particulates [8]. However, particulates can be beneficial in particular applications such as composite systems [10]. Furthermore, there is great interest in the use of pulsed laser ablation for accurate determination of composition [11]. In this case, the potential for non-congruent transfer is of great concern for the accuracy of such techniques.

In this paper, we focus on the non-congruent transfer in the model semiconductor system $\mathrm{SiGe}$ in an attempt to understand the physical processes responsible for this behavior. We find the changes in stoichiometry to be caused by effects that occur in the plume itself rather than at the target or substrate. In addition, we examine the influence of target microstructure on the density and composition of the particulates produced in these films. The particulates in the films maintain the composition of the target and their numbers are significantly reduced by using a denser target.

\section{Experimental Details}

All of the films for this experiment were grown in a high vacuum chamber with a base pressure $<3 \times 10^{-7}$ Torr. Excimer laser light from a Lambda-Physik LPX305i operating on the $\mathrm{KrF}$ line $(\lambda=248 \mathrm{~nm}, 30 \mathrm{~ns})$ was passed into the chamber through a fused silica window and focused onto the target at an angle of $30^{\circ}$ from the surface normal. Our focused spot size was approximately $1 \mathrm{~mm}$ height $\times 7 \mathrm{~mm}$ width and the incident energy on the target was fixed at $360 \mathrm{~mJ} /$ pulse yielding an average fluence of $5 \mathrm{~J} / \mathrm{cm}^{2}$. For all the experiments discussed here, the repetition rate was $2 \mathrm{~Hz}$.

We used two different target preparations: a commercially purchased sintered target and a "homemade" solidified target. The solidified target was made by RF melting pure $(99.9999 \%) \mathrm{Si}$ and $\mathrm{Ge}$ in the appropriate proportion and subsequently quenched to room temperature. The resulting material was then polished flat using 600 grit $\mathrm{SiC}$ paper. Both targets had nominal compositions of 80 at. $\% \mathrm{Si}$ and 20 at. \% Ge (the actual compositions determined by RBS were $18 \% \mathrm{Ge}$ in the sintered target and $21.5 \% \mathrm{Ge}$ in the solidified target). The targets were polished with 600 grit paper between growth runs to refresh their surfaces.

The basic experimental protocol is straightforward. A substrate of glassy carbon approximately $6 \mathrm{~cm}^{2}$ in area is placed $8 \mathrm{~cm}$ from the target on a rotatable mount. A fixed mask with a $0.5 \mathrm{~cm}^{2}$ aperture is positioned directly in front of the substrate. We then ablate the target for a given number of shots. Next, the substrate is rotated to expose a fresh surface behind the mask and subsequent depositions are performed.

For all the results discussed in this paper, the target remains fixed throughout the deposition run. This allows us to count shots at a given location to look at transient behavior in the composition of the resulting films.

We employ three different techniques to analyze the resulting films. The average composition of the films is determined by Rutherford backscattering spectrometry (RBS). Particulates in the films are observed and counted using SEM and their composition is obtained from Electron Microprobe measurements.

\section{Results}

Figure 1 shows the measurement of film composition as a function of cumulative shots on the solidified target. The value on the abscissa is the median shot number for the 
deposition. For example, the data point with an abscissa of 910 represents the average composition for a film deposited during shots 661 through 1160. At low shot numbers, i.e. a fresh target surface, the composition of Ge is very high relative to the target composition. After subsequent shots on the target, the average film composition decreases to what appears to be a steady state composition of $32 \%$ Ge. This steady state result is consistent with the previous work by Antoni et al. [4] who found a $\mathrm{Ge}$ composition between 35 and $40 \%$ for a $20 \%$ Ge target. The results for the sintered target show a similar trend, leading to a steady state composition which is also greater than the target composition.

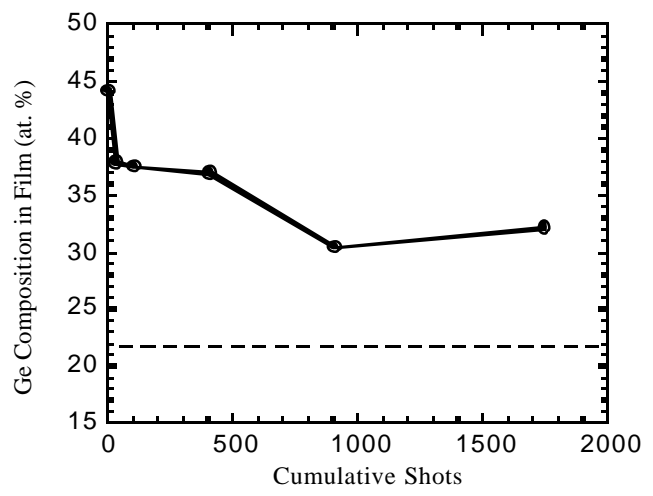

Figure 1. Composition of $\mathrm{Ge}$ in film as a function of the cumulative number of shots on the target. The dashed line denotes the average bulk compostion of the unshot target.

The different explanations for non-stoichiometric behavior reported in the literature can be divided into three main areas: target effects, substrate effects, and plume effects.

\section{$2.1 \quad$ Target effects}

Target effects include preferential ablation from the target [9], thermal evaporation at low energy due to different vapor pressures [6], or segregation occurring at the target surface [4]. The first two effects are unlikely in this experiment since we are dealing with energies well beyond the ablation threshold for Si or Ge. Thus, we expect that both types of atoms would be equally likely to ablate from the surface.

It is well known that upon melting and solidifying $\mathrm{Ge}$ will segregate to the surface of Si [12]. This enrichment was previously observed in the SiGe system [4], and we observe it in our RBS analysis of the target after laser irradiation. However, target segregation itself is not sufficient to describe noncongruent transfer in the steady state, regardless of the system being studied. Intuitively, one realizes that the ablation process removes a volume of material from the target and not merely the topmost surface layer. This volume of material will always have the same composition as the bulk target. We test this idea by using RBS depth profiling and measuring the ablation depth with stylus profilometry. We find the average composition in one ablation depth on modified target is equal to the bulk target composition at high shot number.

The transient behavior we observe at low shot numbers is consistent with target segregation before the steady state is reached. We have modeled this using an analysis similar to that for solute trapping during laser resolidification of alloys
$[12,13]$ with the additional feature of removing material from the upper layers of the simulation target. Surface segregation produces an initially large maximum Ge composition in the topmost layers followed by a decay in maximum composition for subsequent iterations until the steady state is reached [14].

\section{$2.2 \quad$ Substrate effects}

Substrate effects may include sputtering by the incident atoms [6], differences in the sticking coefficients of the species [2], or preferential evaporation from the substrate [7]. Since we are dealing with $\mathrm{Si}$ and $\mathrm{Ge}$ and our substrate remains at the ambient temperature, there should be no evaporation of either species. The typical energies associated with laser ablation make it possible for the incident species to sputter atoms off the substrate. If one type of atom in the substrate is preferentially removed, there will be an overall change in the film composition. In the case of $\mathrm{Si}$ and $\mathrm{Ge}$, the literature values from pure $\mathrm{Si}$ and pure $\mathrm{Ge}$ show that $\mathrm{Ge}$ has a higher sputtering yield than Si and therefore we would expect a film that is Si rich instead of Ge rich. However, it is possible that the presence of multiple species might affect the yield of a component and therefore we check this experimentally.

We probe the possible importance of sputtering at the substrate by changing the incident flux energy when we perform the experiment in an ambient pressure of Ar gas. In order to determine the change in the ion energy, we first measure the energies using a simple time of flight ion probe. At 100 mTorr Ar, the high energy component of the ion signal is severely attenuated. We find no significant changes in the film composition for this case; the steady state composition is still much higher than the target composition. Therefore, we are left to conclude that sputtering effects are not relevant to the nonstoichiometric behavior we see in this system.

Because sticking coefficients are expected to increase toward unity at cryogenic temperatures, we anticipate that differences in the sticking coefficients of depositing species would be more prevalent at higher temperatures than at lower ones. However, since we are dealing with energetic species, these differences may still appear at room temperature. To determine the importance of this effect we cool our substrate to liquid nitrogen temperature by remounting it on a cooled copper block. Upon repeating the experiment with this condition, we still find an enrichment of $\mathrm{Ge}$ in the films. Only slight differences are observed in the initial few shots. This result implies that the $\mathrm{Si}$ is not preferentially scattering off the substrate, but rather that it never gets there. Implicit in this interpretation is that there exists a temperature dependent sticking coefficient even for the energetic deposition associated with PLD.

\subsection{Plume effects}

We are dealing with multiple species which may have different angular distributions in the plume [15]. This can be due to difference in the mass of the species or differences in the charge states of the species [16], or due to the rapid expansion of the plume into the vacuum [17]. We initially tested this idea by looking at the position dependence of the concentration over larger substrate areas. We removed the normal-sized substrate and mask and installed a 3" wafer with pieces of glassy carbon affixed to its face. The resulting composition measurements do not vary significantly across the wafer in either of the vertical or horizontal directions across the substrate. This indicates that the angular distribution for $\mathrm{Si}$ is similar to that for Ge over the 
studied region. It should be noted that the angle subtended in this experiment was approximately $+-15^{\circ}$ in both the vertical and the horizontal directions and therefore does not address the issue of very large angle distributions.

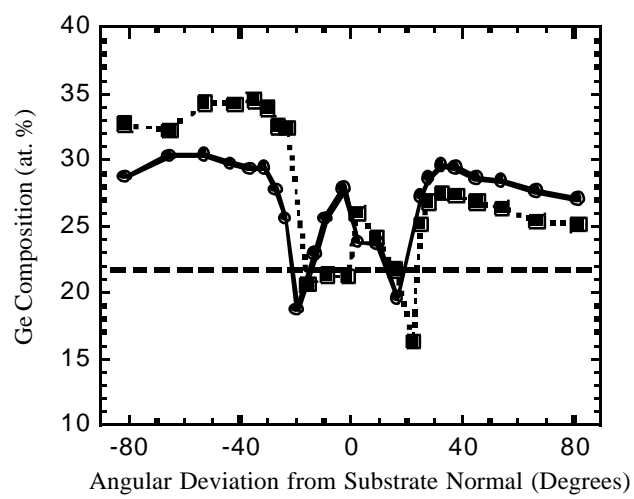

Figure 2. Plot of Ge composition as a function of angle from the center of the substrate after 2000 shots. All these points lie in the vertical plane bisecting the laser spot. The solid line with circles shows the composition distribution in the presence of a substrate. The dotted line with squares shows the same measurement without the substrate. The dashed line represents the bulk composition of the target before laser irradiation. Zero degrees corresponds to the incident laser spot position.

To further study this effect, we collect deposition over all angles within a closed cylinder. The target is covered with a mask so that any material that redeposits on the target surface is also observable. The result of this study is shown in figure 2 . Angular positions are determined from the center of the substrate. The negative values indicate locations on the bottom of the cylinder, and the ablation spot on the target is at an angle of $0^{\circ}$. We can see that as we move closer to the target, the films become less $\mathrm{Ge}$ rich and eventually become $\mathrm{Si}$ rich relative to the initial target. This trend begins to reverse itself along the surface of the target.

We find this result to be unchanged by removing the substrate at one end of the can. Furthermore, it is important to note that even when there is no substrate to backscatter the atoms, material still gets redeposited on the target surface. This is consistent with the analysis of plume expansion in vacuum by Kelly [17].

The increase in Ge composition as we move closer to the laser spot indicates that $\mathrm{Ge}$ is also being backscattered by the plume, but it has a much sharper distribution than the Si. Nonetheless, figure 2 shows evidence that the "missing" Si from the deposited films is being scattered away from the substrate normal and back toward the target surface. Thus we attribute the non-congruent transfer to differential scattering in the plume.

\section{$2.4 \quad$ Particulates}

Figure 3 shows the size distribution of particulates with diameters larger than $1 \mu \mathrm{m}$, determined by SEM imaging for the two target preparations. For the same number of shots, the sintered target clearly produces more particulates than the solidified target. When we integrate the area of these particulates, we find an areal density of $1.6 \%$ for the sintered target and $0.3 \%$ for the solidified target. If we assume all particulates are disk shaped with height $1 \mu \mathrm{m}$, the resulting volumetric fractions of particulates in the films would be $4.3 \%$ and $0.9 \%$ respectively.

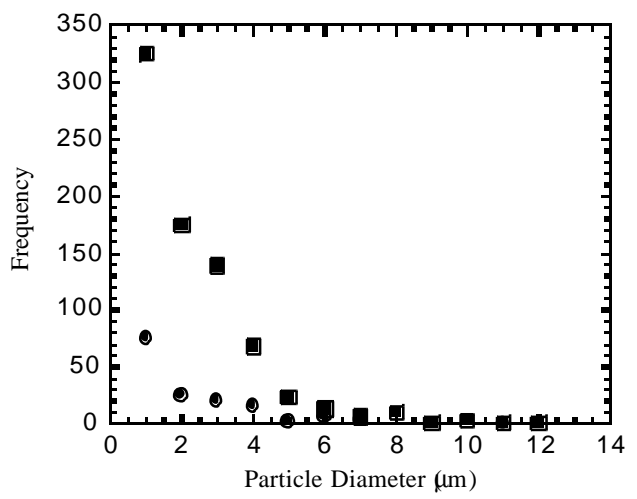

Figure 3. Size distribution of particulates in the deposited films. The filled squares represent the particulates in the films grown from a sintered target and the circles represent the solidified target. These measurements were made on films deposited from shot numbers $161-660$ on the respective targets.

To understand this result, we look carefully at the target surface after laser irradiation. The sintered target shows sharp features on a large length scale while the solidified target appears smoother. Upon higher magnification, the sintered target shows cracks and pits related to the violent ejection of particulates. The solidified target shows ripples with wavelength on the order of the wavelength of the laser.

These results are consistent with results from other systems where sintered targets were compared to solidified targets [18]. The basic explanation is related to the relative density of the targets. For the less dense sintered target, the thermal conductivity is lower. Thus a local irregularity can heat up quickly before transferring the energy to the rest of the bulk target. This leads to a higher local temperature and produces a more explosive event in comparison to the denser target where the heat can be more quickly dissipated to the bulk.

The composition of the particulates is different than the composition of the films. Figure 4 shows the distribution of compositions for all the particles studied. In contrast to the behavior of particulates in metallic systems [10], we did not see any relationship between the size of the particulates and their composition. In addition, we are studying a relatively small area near the center of the substrate in which there does not appear to be any relationship between the position of the particulate and its composition.

In both cases the average particulate composition is approximately the same as the bulk target composition. However, the sintered target produces particulates with a composition distribution that appears broader than that of the solidified target. Although the two distributions have virtually identical standard deviations, the kurtosis $\left(4^{\text {th }}\right.$ central moment) of sintered target data (-0.83) is significantly less than that (2.14) of the solidified target thereby indicating a broader distribution.

These results may be explained by looking at the microstructure of the targets before laser irradiation. The solidified target has relatively large $\mathrm{Si}$ rich grains $(\sim 1-2 \mathrm{~mm})$ with $\mathrm{Ge}$ rich grain boundaries. Thus when the target melts, lateral diffusion will smear out the relatively small grain boundaries, leaving us with a comparably tight distribution of 
compositions. The sintered target has many grains of varying size and composition from nearly pure $\mathrm{Si}$ to nearly pure $\mathrm{Ge}$. In that case, the larger grains or equivalently, large regions where the composition is different than the average, will not mix significantly during the molten state. This allows for a broader distribution of compositions in the resultant particulates.

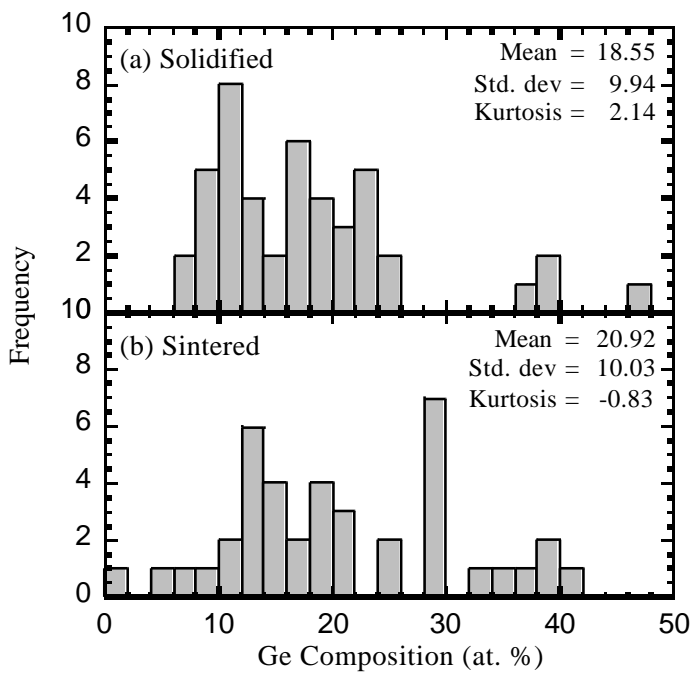

Figure 4. Composition distribution for particulates deposited from (a) solidified and (b) sintered targets. The kurtosis of (a) is 2.14 while that for $(b)$ is -0.83 denoting a sharper distribution for the solidified target. For reference, the kurtosis of a normal distribution is 0 .

\section{Discussions}

The observation that the average composition of particulates is the same as the bulk target is consistent with our analysis for the non-congruent film composition. We have concluded that the non-congruent film composition is due to differential scattering in the plume, which occurs during the rapid expansion of the gaseous species in the plume [17]. However, since the particulates are formed much later in the ablation process by direct ejection of condensed material from the target and move more slowly, we would expect them to be less affected by such scattering events.

The implication of this result is that if one uses a spectroscopic technique on the plume to determine composition of an unknown sample, there could be errors due to differential scattering in the plume and the limited detection angle [18]. However, we have shown that the particulates are not affected by such plume scattering and maintain the target composition. Therefore, provided there is no secondary evaporation during the particulate flight [10], careful chemical analysis of these particulates can provide a technique to measure accurately an unknown target composition.

\section{Conclusions}

We have examined stoichiometric issues involved in PLD of multicomponent $\mathrm{Si} / \mathrm{Ge}$ targets and we find that the target composition is not preserved in the resulting films. The distribution of species reveals the presence of proportionately large amounts of $\mathrm{Si}$ near the target surface, leading to the depletion of $\mathrm{Si}$ in the growing film. We conclude that this effect is due to differential scattering in the plume. Neither segregation of $\mathrm{Ge}$ in the ablation target nor self sputtering has an effect on the steady state composition of the films. However, the transient behavior is consistent with target segregation before the steady state is reached. Additionally, we find that the average composition of particulates in the film maintains the stoichiometry of the bulk target. The influence of target microstructure manifests itself in a sharper distribution of particulate compositions and a lower density of particulates in the films deposited from solidified targets.

\section{Acknowledgements}

The authors thank Dr. T. Yoshida and Dr. Y. Yamada for providing some of our glassy carbon substrates. D. Lange provided guidance and assistance with the Microprobe measurements. In addition, we thank Dr. D. H. Lowndes, Dr. D. B. Geohegan, Dr A. A. Puretsky (sp?), Dr. K. Murakami, Dr. C. M. Rouleau, Dr. J. W. McCamy, Dr. J. Erlebacher, Dr. P. Sanders, and Dr. C. Stauter all for helpful discussions. Research at Harvard was supported by NSF-DMR-97-27369 and in part by the Joint Research Program (1997-1999) of the "Monbusho".

6 References

[1] for example: D. Chrisey and G. Hubler: Pulsed Laser Deposition of Thin Films (Wiley, New York, 1994)

[2] R.P. van Ingen, R.H.J. Fastenau, E.J. Mittemeijer, J. Appl. Phys. 76, 1871 (1994)

[3] H.U. Krebs, S. Fahler, O. Bremert, Appl. Surf. Sci. 86, 86 (1995)

[4] F. Antoni, E. Fogarassy, C. Fuchs, J.J. Grob, B. Prevot, J.P. Stoquert, Appl. Phys. Lett. 67, 2072 (1995)

[5] for example: E. Sobol: Phase Transformations and Ablation in Laser-Treated Solids (Wiley, New York,1995)

[6] E. van de Riet, J.C.S. Kools, J. Dieleman, J. Appl. Phys. 73, 8290 (1993)

[7] D.B. Chrisey, J.S. Horowitz, K.S. Grabowski, Mater. Res. Soc. Symp. Proc. 191, 25 (1990)

[8] E. van de Riet, C.J.C.M. Nillesen, J. Dieleman, J. Appl. Phys. 74, 2008 (1993)

[9] T. Yano, T. Ooie, M. Yoneda, M. Katsumura, J. Mater. Sci. Lett. 15, 1994 (1996)

[10] L.C. Chen, E.L. Hall, Mater. Res. Soc. Symp. Proc. 285, 519 (1993)

[11] C. Nouvellon, C. Chaleard, J.L. Jacour, P. Mauchien, Appl. Surf. Sci. 138, 306 (1999)

[12] D.P. Brunco, M.O. Thompson, D.E. Hoglund, M.J. Aziz, H.J. Gossmann, J. Appl. Phys. 78, (1995)

[13] C.B. Arnold, M.J. Aziz, M. Schwarz, D.M. Herlach, Phys. Rev. B. 59, 334 (1999)

[14] C.B. Arnold, M.J. Aziz, To be published

[15] J. Gonzalo, C.N. Afonso, J. Perriere, R. Gomez San Roman, Appl. Surf. Sci. 96, 693 (1996)

[16] K. Saenger in: D. Chrisey and G. Hubler (eds) Pulsed Laser Deposition of Thin Films (Wiley, NewYork, 1994) p199

[17] R. Kelly, A. Miotello, Nucl. Inst. and Meth. in Phys. Res. B91, $682(1994)$

[18] R.P. van Ingen, PhD. Thesis, Delft University, 1993 